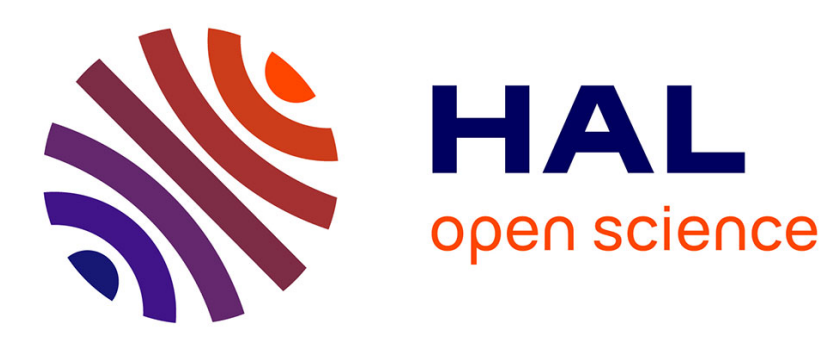

\title{
Impact des interruptions de cotation sur la microstructure du marché boursier français
}

Karine Michalon

\section{To cite this version:}

Karine Michalon. Impact des interruptions de cotation sur la microstructure du marché boursier français. Revue d'économie financière, 2002, 70, pp. 253-259. halshs-00142776

\section{HAL Id: halshs-00142776 \\ https://shs.hal.science/halshs-00142776}

Submitted on 22 Apr 2007

HAL is a multi-disciplinary open access archive for the deposit and dissemination of scientific research documents, whether they are published or not. The documents may come from teaching and research institutions in France or abroad, or from public or private research centers.
L'archive ouverte pluridisciplinaire HAL, est destinée au dépôt et à la diffusion de documents scientifiques de niveau recherche, publiés ou non, émanant des établissements d'enseignement et de recherche français ou étrangers, des laboratoires publics ou privés. 


\title{
IMPACT DES INTERRUPTIONS DE COTATION SUR LA MICROSTRUCTURE DU MARCHE BOURSIER FRANÇAIS
}

Novembre 2002

\section{Résumé}

Les interruptions de cotation réglementées d'Euronext Paris et de toutes les autres places boursières ont pour but de réduire les asymétries d'informations et la volatilité et de permettre l'émergence d'un consensus. L'usage de ces interruptions est très fréquent sur le marché boursier français des valeurs qui composent le CAC 40, le SBF 120 et le SBF 250: on constate en moyenne plus de 7 interruptions par séance. La fréquence est d'autant plus élevée que les valeurs ont de faibles capitalisations boursières. Pourtant notre étude empirique fait ressortir une inefficacité des réservations de cotation. En effet, la volatilité des cours est plus élevée après réservation.

Mots clés : coupe-circuit, réservation, suspension, volatilité

Impact of the interruptions of quotation on the microstructure of the French stock exchange market

\begin{abstract}
The purpose of the regulated interruptions of quotation on Stock exchange markets is to reduce asymmetries of information and volatility and to allow the emergence of a consensus. The use of these interruptions is very frequent on the French stock exchange market for the values included in the CAC 40, the SBF 120 and the SBF 250 indexes : one notes on average more than 7 interruptions per trading day. The frequency is all the more high as the values have weak market capitalizations. However, our empirical study emphasizes an inefficiency of the reservations of quotation. Indeed, the volatility of the prices is higher after reservation.
\end{abstract}

Keywords : circuit breaker, trading halt, volatility

JEL : C2, G10 


\section{Introduction}

Aujourd'hui, les réglementations de toutes les places boursières prévoient des procédures d'interruption de séances de cotation. Le terme générique interruption de cotation regroupe tout arrêt de l'échange sur la bourse, autre que la période définie par l'inter-séance qui s'étend de l'heure de la clôture d'une séance à l'ouverture du lendemain. Le rôle annoncé des interruptions de cotation réglementées est de permettre l'information des participants et de protéger les intérêts des petits porteurs. Il s'agit donc de réduire les asymétries d'informations et la volatilité, de permettre l'émergence d'un consensus. Les modalités d'arrêt des cotations peuvent être individuelles ou collectives. Dans cet article, nous ne nous intéresserons qu' aux interruptions de cotation exceptionnelles à seuil de déclenchement individuel du marché boursier français des actions. Ce sont des mesures d'interruption des cotations prises au niveau d'un seul titre. Ce type de réglementation est incontestablement le plus ancien et le plus universel. Ces interruptions sont de deux types. Elles peuvent prendre soit la forme de réservations, soit celle de suspensions. Les réservations ont lieu sur un titre quand son cours approche une certaine limite appelée seuil de réservation. Les suspensions sont exceptionnelles et concomitantes à des événements spéciaux surgissant dans la vie de la société émettrice.

Dans un tel contexte, il apparaît intéressant d'étudier l'impact de ces ruptures de cotation sur le comportement des agents et les processus de prix, objets d'étude de la microstructure. Tel est le but de cet article

Dans une première section, nous verrons comment la bourse de Paris définit les modalités d'arrêt des cotations. Nous procéderons également à une brève revue de littérature sur l'impact des interruptions de cotation. Dans une deuxième section, nous étudierons les interruptions de cotation d'un point de vue empirique en considérant le cas du marché boursier français. Nous tenterons ici d'apporter des réponses à diverses interrogations : quelle est la fréquence des interruptions de cotation? Dans quelle mesure les réservations de cotation atteignent les objectifs qui leurs sont assignés? Quel est leur effet réel sur la volatilité ? 


\section{Les interruptions de cotation exceptionnelles à seuil de déclenchement individuel}

Nous commencerons par donner quelques définitions générales liées aux réservations et aux suspensions. Nous mettrons ensuite en évidence les effets contradictoires des interruptions de cotation en dressant une brève revue de littérature.

\subsection{Réservations et suspensions à Paris}

Dans le cadre de la mise en place du Modèle de Marché d'Euronext, les marchés actions d'Euronext ont été modifiés dans leur fonctionnement, notamment en termes d'horaires de cotation et de modalités de réservation. Nous présentons ici les nouvelles règles de cotations, en application depuis le 23 avril 2001.

\subsubsection{Les réservations}

Une réservation est un arrêt des cotations en cas de décalage de cours important par rapport à la clôture précédente. Les modalités exactes sont déterminées en fonction du mode de cotation.

\subsubsection{Les modes de cotation}

Selon le principe général du cycle de négociation (article 4301 du livre I des règles de marché d'Euronext Paris), « les titres se négocient soit par appariement continu des ordres de sens opposé dans le carnet central, soit par fixing, c'est à dire confrontation générale des ordres après une période d'accumulation sans exécution. La répartition des titres entre les modes continu et fixing est déterminée par l'entreprise de Marché d'Euronext Compétente sur la base de critères objectifs tels que, de manière non limitative, les volumes historiques ou anticipés, l'appartenance à un indice d'Euronext ou un autre indice reconnu internationalement, et la présence d'Apporteurs de liquidité. » Le déroulement de la séance est différent suivant le mode de cotation : 
- Pour les valeurs négociées en continu :

- de 7 h 15 à 9 h 00, pré-ouverture, les ordres s'accumulent sans qu'aucune transaction n'intervienne,

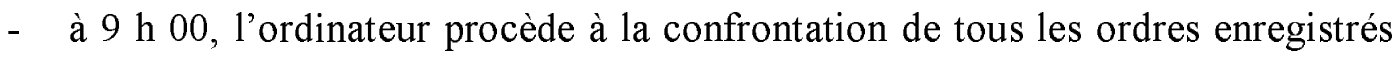
et détermine le prix d'équilibre - ou fixing d'ouverture - qui permet l'échange du plus grand nombre de titres,

- de 9 h 01 à 17 h 25, le marché fonctionne en continu,

- de 17 h 25 à 17 h 30, pré-clôture : les ordres s'accumulent sans transaction comme pendant la pré-ouverture,

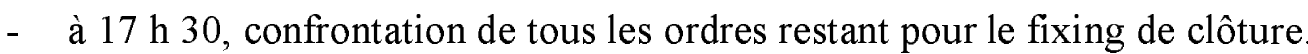

- Pour les valeurs négociées en fixing :

- de 7 h 15 à 10 h 30 : pré-ouverture,

- 10 h 30 : fixing d'ouverture,

- de 10 h 30 à 16 h 00 : pré-clôture,

- 16 h 00 : fixing de clôture (les valeurs du Marché Libre d'Euronext Paris font l'objet d'un fixing quotidien unique à 15 h 00).

\subsubsection{Les règles de réservation}

Les réservations résultent de l'impossibilité momentanée d'ajuster l'offre et la demande à l'intérieur des zones de cours autorisés. Les modalités pratiques d'application actuellement en vigueur datent du 23 avril 2001. En effet, Euronext réserve temporairement la cotation d'une valeur dès lors qu'un ordre d'achat ou de vente provoquerait, s'il était exécuté, des négociations à un (ou des) cours situé en dehors des limites appelées «seuil de réservation ». Les seuils de réservation sont établis en appliquant un pourcentage de fluctuation maximale à un cours dit « cours de référence ».

- Les seuils statiques de réservation

Ils s'appliquent à toutes les valeurs. Ils sont de + ou $-10 \%$ calculés par rapport à un cours de référence statique. A l'ouverture de la séance, le cours de référence statique est le dernier cours coté (en général, le cours de clôture la veille, ajusté si nécessaire en cas de détachement de dividende ou de toute autre opération) ou le dernier cours indicatif diffusé. 
- Si le cours d'ouverture s'établit à l'intérieur de la zone autorisée, le cours d'ouverture devient le nouveau cours de référence statique à partir duquel s'établissent les nouveaux seuils qui ne peuvent être franchis sans provoquer une réservation. (Pour les valeurs cotées en fixing seulement, le cours du fixing d'ouverture devient le cours de référence pour le fixing de clôture).

- Si le cours d'ouverture ne peut s'établir à l'intérieur de ces seuils, la valeur est réservée jusqu'au prochain fixing pour les valeurs cotées en fixing; pour les valeurs cotées en continu, Euronext opère une réservation de 4 minutes après une période de gel qui n'aura pas excédé 1 minute et le seuil atteint devient le nouveau cours de référence.

Il n'existe plus de limite au nombre de réservations possibles au cours d'une même séance. Toutefois, après qu'une valeur ait franchi deux seuils statiques, Euronext se réserve la possibilité de proroger la période de réservation au vu de l'état du carnet d'ordres.

- Les seuils dynamiques de réservation

Ils ne s'appliquent qu'en séance aux valeurs cotées en continu et visent à encadrer, à l'intérieur des seuils statiques, la volatilité des cours successifs.

Chaque cours coté devient le cours de référence dynamique pour déterminer les seuils maximum et minimum à ne pas dépasser pour le cours suivant. Les seuils dynamiques sont de + ou $-2 \%$ pour les actions les plus liquides, dont les valeurs du CAC 40 , et à + ou $-5 \%$ pour les autres par rapport au cours de référence dynamique. Ainsi un cours d'ouverture qui se situe à l'intérieur des seuils autorisés détermine les nouveaux seuils statiques de réservation et les seuils dynamiques qui encadrent le prix d'exécution de la prochaine transaction.

Trois cas de figures sont alors possibles:

1. la prochaine transaction s'effectue à l'intérieur des seuils dynamiques; le cours de cette transaction devient le nouveau cours de référence dynamique et ainsi de suite sous réserve de ne pas dépasser les seuils statiques.

2. la prochaine transaction s'effectue intégralement en dehors des seuils. La valeur est « gelée » et le négociateur qui a introduit l'ordre dispose d'une minute pour :

- confirmer son ordre, ce qui entraîne la réservation de la valeur 4 minutes, 
- modifier son ordre, pour le positionner à l'intérieur des seuils, ce qui permet la reprise des négociations sans réservation.

A défaut, à la fin du gel, l'ordre est rejeté et les négociations reprennent.

3. la prochaine transaction s'effectue pour partie à l'intérieur et pour partie à l'extérieur des seuils : hormis le cas d'un ordre «à tout prix» ou d'un ordre limité « tout ou rien », l'ordre est exécuté pour la partie à l'intérieur des seuils et la réservation de la valeur est déclenchée pour la partie non exécutée

Les seuils de réservation dynamiques ont notamment pour objet de prévenir les conséquences néfastes de possibles erreurs de saisie.

Pendant la réservation, les valeurs sont en phase de pré-ouverture ; il est possible de rentrer des ordres sur la feuille de marché mais aucune transaction n'intervient et la cotation reprend par un fixing comme à l'ouverture.

\subsubsection{Les suspensions à Paris}

Elles résultent d'une décision d'Euronext et font l'objet d'un avis indiquant :

1. l'origine : la suspension peut intervenir à la demande des régulateurs de marché ( $\mathrm{CMF}$ ou $\mathrm{COB})$, de la société ou sur l'initiative d'Euronext ;

2. les raisons (dépôt d'un projet d'offre, attente d'un communiqué...);

3. la date et les conditions de reprise des cotations; à défaut l'avis précise que la valeur reste suspendue jusqu'à nouvel avis.

La suspension de cotation d'une valeur entraîne l'élimination des ordres non-exécutés sur cette valeur, au contraire de la réservation.

Le but est de protéger la masse des épargnants en évitant:

- Que, dans une période troublée de la vie d'une entreprise, des personnes disposant d'informations privilégiées puissent en profiter en Bourse 
- Qu'au moment où la situation d'une affaire peut basculer complètement, des actionnaires achètent ou vendent un titre dont la valeur peut être radicalement modifiée.

\subsection{Brève revue de littérature}

Une zone de perturbation accompagne le changement de niveau d'équilibre, lors de l'arrivée d'une information. Le passage vers le nouveau niveau d'équilibre est-il facilité par une interruption des cotations? Ou au contraire, la continuité des cotations permet-elle d'absorber plus rapidement le choc ?

La littérature théorique est contradictoire : dans certains modèles la suspension facilite la dissémination des informations et réduit la volatilité. En effet, à la suite du Krach de 1987, un certain nombre d'analystes au NYSE, incluant le corps expéditionnaire présidentiel spécialisé sur les mécanismes du marché, ont recommandé l'établissement du mécanisme « circuit breaker ». Le premier argument en faveur des mécanismes « coupe circuit » est que les périodes de non échange fournissent une opportunité pour la transmission normale des informations dans les délais du marché. Les partisans des «coupe circuit» pensent que pendant les mouvement importants de prix, il peut y avoir une rupture dans la transmission de l'information entre la salle de marchés et les participants. Par conséquent, « la première fonction d'une procédure de «coupe circuit» devrait être de réinformer les participants » (Greenwald et Stein (1988), p 17). En diminuant les asymétries d'informations entre les traders, les suspensions permettraient l'émergence d'un nouveau consensus de prix.

Dans d'autres modèles, la cotation en continu est préférable pour la découverte des prix. Plusieurs arguments corroborent cette thèse. En effet, lors de la réouverture, l'intérêt des investisseurs n'est pas forcément de révéler immédiatement l'information qu'ils détiennent, ce qui peut conduire à un bruitage temporaire des prix. Par ailleurs, l'accroissement de la couverture médiatique de la réservation amène de nouveaux investisseurs et peut être un facteur d'éclatement de consensus, de dispersion accrue des anticipations et donc d'accroissement de la volatilité !

Beaucoup de théoriciens doutent ouvertement d'une quelconque interférence du marché et pensent que les suspensions d'échanges en seraient au contraire responsable. Grossman (1990, p.3), par exemple expose que la fermeture des marchés «empêche simplement des adultes consentants d'exécuter leur désir dans les salles de marché de la bourse ». Non seulement faire des suspensions entraîne un coût de liquidité pour les traders, mais les études suggèrent que l'information ne sera pas aussi bien révélée pendant une suspension que 
pendant une séance de cotation en continu (Miller(1990), McMillan (1990), Grundy and McNichols (1989), Brown and Jennings (1989), Dow et Gorton (1989)).

Nous avons choisi de centrer notre exposé sur deux études empiriques : d'une part, l'étude de Lee, Ready et Seguin (1994) examinant l'impact des suspensions sur la volatilité et le volume des cours au NYSE et d'autre part, l'étude de Jouaber (2000) portant sur le marché français.

\subsubsection{Etude de Lee, Ready et Seguin - la cotation en continu est préférable pour la découverte des prix}

Lee, Ready et Seguin (1994) étudient l'efficacité des haltes en examinant l'effet des suspensions sur le volume des échanges et la volatilité des prix d'entreprises particulières du NYSE. L'échantillon initial est constitué de toutes les suspensions de séance du NYSE durant l'année 1988 enregistrées par l'ISSM, soient 852 suspensions d'échanges de trois types : les suspensions dues à des déséquilibres importants constatés entre l'offre et la demande d'actions, à des informations en cours de diffusion et à des informations attendues. Ils ont comparé les statistiques d'une suspension et celles provenant des 6.5 heures suivant la réouverture des échanges à celles provenant d'une pseudo-halte : une période de cotation en continu commençant au même moment de la journée et de même durée, avec des variations de cours de même ampleur (à $1 \%$ près) ; ce qui réduit l'échantillon à 500 couples de haltes et de pseudo-haltes. Cette procédure élimine les événements les plus «dramatiques »en terme d'impact sur les prix. L'échange de réouverture qui termine la suspension n'a pas un volume significativement plus élevé que le volume cumulé pendant la période de pseudo-halte. Ces résultats suggèrent que si les traders sont en clin à minimiser leur demande pendant la suspension, cette activité n'est pas reflétée dans le niveau du volume de réouverture. Mais, le volume et la volatilité dans les 6.5 heures qui suivent la suspension sont significativement plus importants que les mesures correspondantes suivant la pseudo-halte. En effet pour les 500 observations, le volume sur le reste de la séance à partir de la réouverture est $230 \%$ plus élevé que la normale. Les changements absolus de prix et l'estimateur des valeurs extrêmes sont tous les deux 50\% plus élevés que la normale et le nombre de corrections de cotations est $117 \%$ plus élevé que la normale. Lee, Ready et Seguin montrent qu'une partie substantielle du volume et de la volatilité post-suspension est associée à l'étendue de la couverture médiatique. La plupart des suspensions de cotation reçoivent une attention médiatique, alors 
qu'uniquement la moitié des pseudo-haltes est associée à des annonces de nouvelles économiques. De plus, le volume et la volatilité ne sont pas significativement importants après les suspensions non liées à des annonces. La couverture médiatique pourrait être un facteur explicatif des résultats post-suspension. Un autre facteur potentiel pour expliquer la partie restante est la classe de modèles «learning-by-trading». Etant donné qu'une interruption de cotation suspend le flux des transactions récentes, il est possible que la perturbation de cette importante source d'information gêne le processus d'ajustement des prix. Les agents ne sont pas disposés et ne peuvent pas révéler leur demande. En conséquence, les prix sont des estimateurs biaisés des valeurs d'équilibre. Le mouvement important dans le volume et la volatilité post-suspension dans la demi heure après la halte et la rapide diminution des mesures de la volatilité par la suite sont cohérents avec cette hypothèse. Cependant, à moins que le marché soit extrêmement lent à apprendre en échangeant, les hauts volumes persistant à $\mathrm{j}+2$ et $\mathrm{j}+3$ ne semblent pas être expliqués par ces modèles.

Les résultats présentés dans cette étude suggèrent donc que les suspensions de cotation ne « calment » pas le marché.

\subsubsection{Etude de Jouaber}

Jouaber (2000) étudie de façon empirique l'efficacité ou non des réservations sur le marché boursier français des actions. L'échantillon est constitué de 109 réservations pour 47 titres du SBF 250 cotés en continu entre juillet 1991 et mars 1994. Les résultats montrent un accroissement de la volatilité et du volume durant les heures précédant la réservation de cotation. Après la reprise, une baisse progressive de la volatilité, qui reste toutefois à un niveau élevé, est accompagnée par une augmentation du volume. L'accélération du nombre de transactions restent observables jusqu'à deux séances après la reprise.

Malgré l'observation de la baisse de la volatilité après la reprise de cotation, l'efficacité des réservations ne peut être affirmée. D’abord, le niveau de la volatilité reste élevé après la fin de la période d'interruption, ensuite l'accroissement de la volatilité observé avant l'interruption peut être la conséquence d'une anticipation de la réservation sur le marché. En effet, la baisse de la volatilité observée après la reprise peut être interprétée de deux manières différentes : une première interprétation consiste à dire que la réservation de cotation est le résultat d'un excès de volatilité et qu'interrompre la cotation engendre une réduction de cette volatilité. Une autre interprétation consiste à appréhender la forte volatilité observée avant la réservation comme le résultat de l'anticipation par certains agents de l'interruption prochaine des 
cotations. Si les niveaux élevés de volatilité observés sur les séances après la reprise rendent la première hypothèse moins vraisemblable, une étude de la probabilité associée à la réservation permet éventuellement de corroborer l'hypothèse d'anticipation.

Lee, ready et Seguin (1994) ont trouvé des résultats similaires à ceux de Jouaber (200) à la Bourse de Paris, pour le volume. Les résultats sont toutefois différents pour la volatilité. La divergence des résultats pourraient provenir de la spécificité des procédures d'interruptions sur les deux places. Néanmoins, globalement, d'après les deux études, il y inefficacité des interruptions en terme de volatilité. Nous allons maintenant procéder à notre propre application empirique sur le marché français.

\section{Etude empirique des réservations à la bourse de Paris}

Cette section a pour objet d'examiner les interruptions de cotation d'un point de vue empirique. Après avoir présenté les données utilisées, nous examinerons la fréquence des interruptions de cotation et enfin, nous étudierons l'impact des réservations de cotation sur la microstructure.

\subsection{Présentation des données}

Notre étude porte sur le mois de février $1998^{1}$. Les données sur les interruptions de cotation pratiquées sur le marché dont issues des CDROM de données intraquotidiennes de ParisBourse $^{\mathrm{SBF}}$. Plus précisément, nous avons utilisé :

- La table BDM3D2 : fichier d'événements de cotation

Dans ce fichier, un indicateur de l'état de cotation de la valeur est associé à chaque observation. La valeur de cet indicateur précise si la cotation de la valeur est suspendue, réservée à la hausse, réservée à la baisse et, dans le cas où il y aurait eu suspension ou réservation, s'il y a eu reprise de la cotation le jour même. En outre, les interruptions de cotation se distinguent par leur origine qui peut être manuelle ou automatique ${ }^{2}$. Chaque suspension, réservation à la hausse, réservation à la baisse ou réservation simple (existence

\footnotetext{
${ }^{1}$ En 1998, la réglementation des réservations était différente de celle qui a fait suite à la création d'Euronext.

${ }^{2}$ Une erreur de transmission ou une erreur de saisie d'un ordre peuvent de manière non désirée provoquer des variations importantes de cours. Dans une première étape, une confirmation est demandée par ParisBourse ${ }^{\mathrm{SBF}}$ au donneur d'ordre et dans l'attente de la réponse, la réservation est manuelle et la marché non informé de l'interruption.
} 
d'ordres au mieux sur une valeur dont le cours théorique d'ouverture ne peut être déterminé), est signalée dans la base de données horodatées de la SBF par respectivement un « $\mathrm{S}$ », un « $\mathrm{H} »$, un « $\mathrm{B} »$ ou un $« \mathrm{P} »$.

- La table BDM1D2 : fichier des transactions

- La table BDVAD2 : fichier type de valeur

- La table BDCCD2 : fichier caractéristique de cotation d'une valeur.

Notre échantillon initial est ainsi formé de toutes les interruptions de cotation constatées en février 1998. Nous avons alors calculé le nombre d'interruptions de cotation par entreprises. La section suivante présente les résultats de cette étude.

\subsection{Statistiques sur la fréquence des interruptions}

Nous avons choisi de nous limiter aux entreprises du CAC 40, du SBF120 et du SBF250. Dans un premier temps, nous présentons ici le nombre d'interruptions de cotations par entreprises. Les résultats sont classés par capitalisations boursières croissantes. Les capitalisations (en millions d'euros) sont extraites de la base de données DATASTREAM.

Tableau 1 : valeurs appartenant à l'indice CAC 40

\begin{tabular}{lllllll}
\hline & $\begin{array}{l}\text { Capitalisations } \\
\text { boursières }\end{array}$ & $\begin{array}{l}\text { Réservations } \\
\text { à la hausse }\end{array}$ & $\begin{array}{l}\text { Réservations } \\
\text { à la baisse }\end{array}$ & $\begin{array}{l}\text { Réservations } \\
\text { simples }\end{array}$ & $\begin{array}{l}\text { Réservations } \\
\text { totales }\end{array}$ & Suspensions \\
\hline $\begin{array}{l}\text { CAP } \\
\text { GEMINI }\end{array}$ & 5811 & 0 & 1 & 0 & 1 & 0 \\
\hline AGF & 8568 & 0 & 1 & 0 & 1 & 0 \\
\hline
\end{tabular}

Tableau 2: valeurs appartenant à l'indice SBF 120 mais pas à l'indice CAC 40

\begin{tabular}{|c|c|c|c|c|c|c|}
\hline & $\begin{array}{l}\text { Capitalisations } \\
\text { boursières }\end{array}$ & $\begin{array}{l}\text { Réservations } \\
\text { à la hausse }\end{array}$ & $\begin{array}{l}\text { Réservations } \\
\text { à la baisse }\end{array}$ & $\begin{array}{l}\text { Réservations } \\
\text { simples }\end{array}$ & $\begin{array}{l}\text { Réservations } \\
\text { totales }\end{array}$ & Suspensions \\
\hline$\overline{\text { SOPHIA }}$ & 138 & 1 & $\overline{1}$ & 0 & 2 & 0 \\
\hline BAIL-INVEST. & 396 & 0 & 1 & 0 & 1 & 0 \\
\hline$\overline{\text { SAT }}$ & 545 & 4 & 1 & 0 & 5 & 1 \\
\hline REMY COINTREAU & 599 & 0 & 1 & 0 & 1 & 0 \\
\hline ALTRAN TECHN. & 871 & 3 & 1 & 0 & 4 & 0 \\
\hline UNIBAIL & 956 & 1 & 0 & 0 & 1 & 0 \\
\hline SALOMON & 1385 & 0 & 0 & 0 & 0 & 9 \\
\hline COFLEXIP & 1477 & 1 & 0 & 0 & 1 & 0 \\
\hline MACHINES BULL & 1522 & 0 & 1 & 0 & 1 & 0 \\
\hline SAGEM & 1569 & 0 & 1 & 0 & 1 & 1 \\
\hline SEITA & 1940 & 1 & 1 & 0 & 2 & 0 \\
\hline
\end{tabular}


Tableau 3: valeurs appartenant à l'indice SBF 250 mais pas à l'indice SBF 120

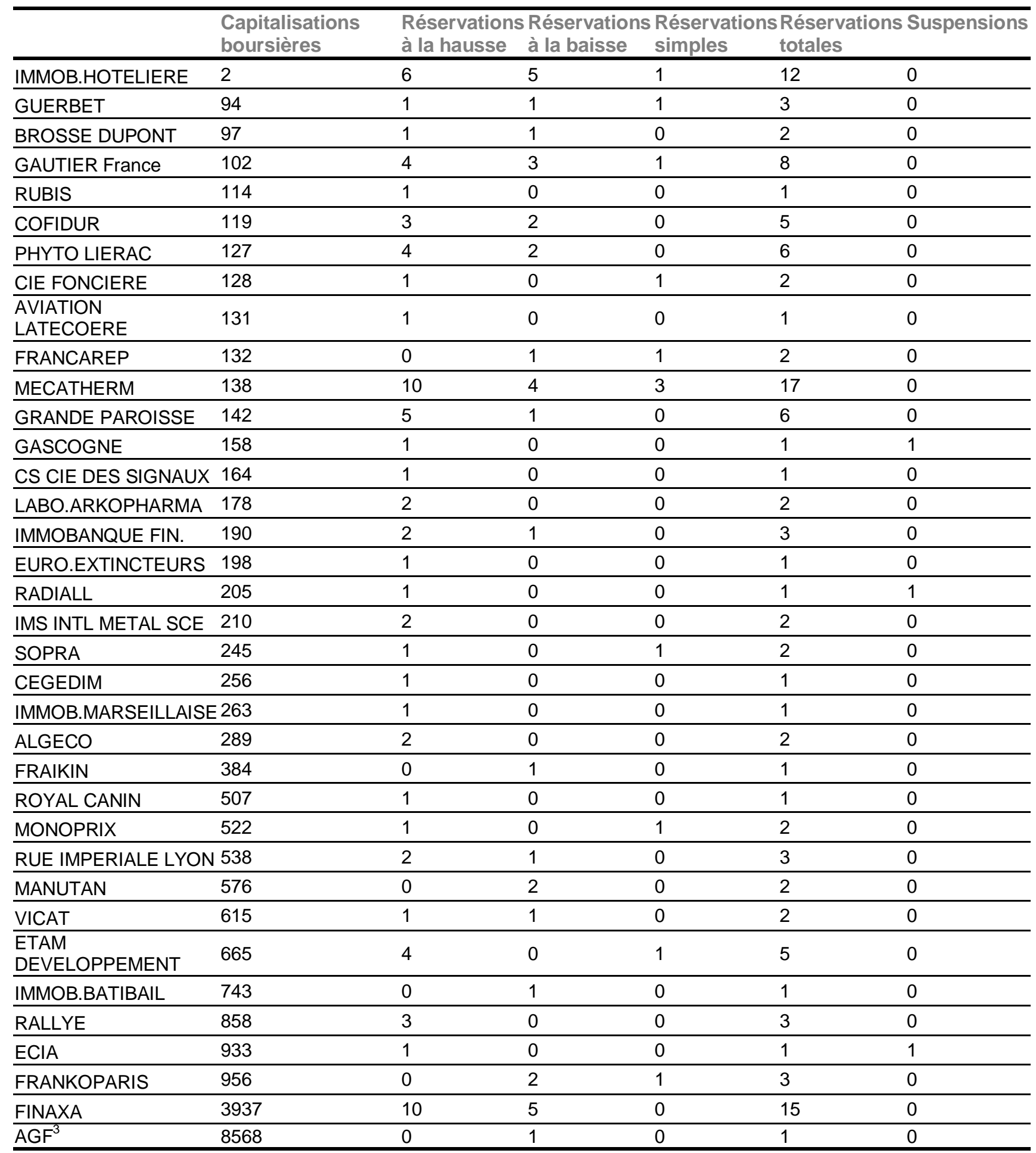

On constate que 48 entreprises sont sujettes aux interruptions de cotation en février 1998. Il y a 157 interruptions de cotations dont 87 réservations à la hausse, 44 réservations à

${ }^{3}$ Les actions AGF ont été retirées provisoirement des échantillons du CAC40 et du SBF120 à partir du 26 février 1998. 
la baisse, 12 réservations simples et 14 suspensions. En moyenne en février 1998, un titre a connu 3.27 interruptions de cotations (2.98 réservations et 0.29 suspensions). C'est MECATHERM qui connaît le plus de réservations (17 réservations). En février 1998 (20 séances de bourse), il y a en moyenne 7.85 interruptions de cotation par séance (7.15 réservations et 0.70 suspensions).

On remarque que les entreprises du SBF250 ont été plus sujettes aux interruptions de cotation en février 1998. Comme dans l'étude de Jouaber (2000), nous pouvons conclure que les sociétés avec de petites capitalisations sont plus exposées aux interruptions de cotation. En effet, 40 entreprises (83\%) des valeurs sujettes aux interruptions de cotation sont de petites capitalisations boursières (dont la valeur boursière est inférieure à 1 milliard d'euros). Les grandes capitalisations boursières (dont la valeur boursière est supérieur à 5 milliards d'euros) ne représentent que $4 \%$ de notre échantillon de sociétés.

\subsection{Impact des réservations sur la volatilité}

Nous avons choisi de nous limiter à l'étude des réservations pour une raison de disponibilité des données. « De plus, la fréquence de leur activation sur la Bourse de Paris, rend opportun de vérifier leur utilité. » (Jouaber (2000)).

Nous avons envisagé dans un premier temps que les réservations puissent être liées à un certain type d'événement (3.3.1). Dans un deuxième temps, nous avons étudié l'impact des réservations sur la volatilité des cours (3.3.2).

\subsubsection{Les possibles évènements sous jacents aux réservations}

Il paraît intéressant de déterminer si les réservations sont liées à un certain type d'événement. Dans ce cas, il est possible d'effectuer une classification des réservations par type d'événement sous-jacent. Nous avons donc utilisé la base de données des ECHOS et les Décisions et Avis de la SBF pour tenter d'identifier les informations ayant causé la réservation. La recherche n'a pas été très fructueuse. Des informations ont cependant été trouvées pour les entreprises suivantes grâce à la base de données des ECHOS :

- Un événement sous-jacent à la réservation du 25/02/1998 de SAT et de SAGEM : fusion. 
- Un événement sous-jacent à la réservation du 26/02/1998 d'AGF : sortie du CAC40 et du SBF120.

- Un événement sous-jacent à la réservation du 20/02/1998 d'IMS : annonce de résultat.

- Une information sous-jacente à la réservation du 17/02/1998 d'IMMOBILIERE HOTELIERE : remboursement d'une créance.

- Une information sous-jacente à la réservation du 26/02/1998 de RALLYE : conséquence d'une offre publique de Rallye sur Casino.

Ceci n'est cependant pas représentatif et l'on ne peut pas réellement en tirer de conclusions.

\subsubsection{Impact des réservations sur la microstructure}

\subsubsection{Echantillon d'entreprises}

Nous excluons un certain nombre de cas de l'étude car ils ne permettent pas d'examiner l'évolution de la volatilité avant et après la réservation :

- Toutes les entreprises dont la cotation du titre est interrompue en début ou en fin de mois.

\begin{tabular}{||l|l||}
\hline \multicolumn{1}{|c|}{ ENTREPRISES } & \multicolumn{1}{c||}{$\begin{array}{c}\text { DATES DE } \\
\text { RESERVATIONS }\end{array}$} \\
\hline AGF & 26 \\
\hline ALGECO & 5 \\
\hline AVIATION LATECOERE & 9 \\
\hline COFLEXIP & 5 \\
\hline FRAIKIN & 3 \\
\hline GUERBET & $4,5,6$ \\
\hline IMMOB. BATIBAIL & 5 \\
\hline IMMOB. MARSEILLAISE & 24 \\
\hline IMS INTL METAL SCE & 20 \\
\hline LABO. ARKOPHARMA & 27 \\
\hline MONOPRIX & 3,4 \\
\hline RUBIS & 27 \\
\hline SAGEM & 25 \\
\hline SAT & 25 \\
\hline SEITA & 23 \\
\hline SOPRA & 19,20 \\
\hline UNIBAIL & 5 \\
\hline \hline
\end{tabular}

- Les entreprises dont le titre est réservé de nombreuses fois au cours du mois

\begin{tabular}{||l|l||}
\hline \multicolumn{1}{|c|}{ ENTREPRISES } & \multicolumn{1}{c|}{$\begin{array}{c}\text { DATES DE } \\
\text { RESERVATIONS }\end{array}$} \\
\hline CIE FONCIERE & 2,20 \\
\hline COFIDUR & 20,24 \\
\hline ETAM DEVELOPPEMENT & $6,11,12,13$ \\
\hline FINAXA & $2,6,9,16,17,20,23$ \\
\hline FRANCAREP & 25,26 \\
\hline
\end{tabular}




\begin{tabular}{||l|l||}
\hline FRANKOPARIS & $5,9,10$ \\
\hline GAUTIER France & $11,12,13,18$ \\
\hline GRANDE PAROISSE & 2,5 \\
\hline IMMOBANQUE FIN. & 17,23 \\
\hline IMMOBILIERE HOTELIERE & $2,3,4,17,18$ \\
\hline MECATHERM & $3,4,9,10,13,16$ \\
\hline PHYTO LIERAC & $9,23,24$ \\
\hline RALLYE & $5,11,26$ \\
\hline RUE IMPERIALE LYON & 5,25 \\
\hline
\end{tabular}

- Les entreprises dont la cotation du titre est réservée puis suspendue: GASCOGNE, RADIALL, ECIA

A l'issue de ces opérations, nous conservons 13 entreprises. Cependant, la totalité des cours du mois n'est présente que pour sept entreprises sur le CD de février :

\begin{tabular}{||l|l|l||}
\hline \multicolumn{1}{|c|}{ ENTREPRISES } & $\begin{array}{c}\text { DATES DE } \\
\text { RESERVATION }\end{array}$ & $\begin{array}{c}\text { TYPE DE } \\
\text { RESERVATION }\end{array}$ \\
\hline ALTRAN TECHNOLOGIE & 13 & $\begin{array}{l}3 \text { à la hausse } \\
\text { à la baisse }\end{array}$ \\
\hline BROSSE DUPONT & 12 & $\begin{array}{l}1 \text { à la hausse } \\
\text { la a la baisse }\end{array}$ \\
\hline CEGEDIM & 17 & 1 à la hausse \\
\hline EURO. EXTINCTEURS & 19 & 1 à la hausse \\
\hline MANUTAN & 16 et 17 & 2 à la baisse \\
\hline ROYAL CANIN & 19 & 1 à la hausse \\
\hline VICAT & 10 & $\begin{array}{l}1 \text { à la hausse } \\
\text { la la baisse }\end{array}$ \\
\hline \hline
\end{tabular}

Nous retiendrons donc finalement ces sept entreprises.

\subsubsection{Méthodologie}

Avant de déterminer la volatilité journalière, il est nécessaire de calculer les rentabilités et donc de régulariser les données. A cette fin, nous avons adopté la démarche suivante :

1. faire la moyenne des prix ayant lieu à la même date heure minute seconde et la somme des volumes

2. régulariser les données toutes les heures

3. calculer la rentabilité comme la variation logarithmique des prix

4. calculer l'écart-type des rentabilités sur la journée 


\subsubsection{Résultats}

Le but de cette étude est d'étudier la volatilité et le volume des cours avant et après la réservation. Nous avons dans un premier temps considéré les entreprises pour lesquelles nous disposions des cours du lundi 2 février au vendredi 27 février $1998^{4}$. Il s'agit des 7 entreprises précédemment citées. Parmi elles, la fréquence des transactions est telle qu'il n'a été possible de calculer des volatilités quotidiennes tous les jours ouvrés du mois que pour ALTRAN TECHNOLOGIE et ROYAL CANIN.

Pour pouvoir tirer des conclusions ou avoir des intuitions (la petitesse de l'échantillon fait qu'on ne peut pas faire de déductions générales), nous avons néanmoins examiné les résultats d'EURO EXTINCTEURS (16 volatilités sur 20) et ceux des 6 entreprises dont les cours sur le CD ne sont disponibles que jusqu' au 20 février.

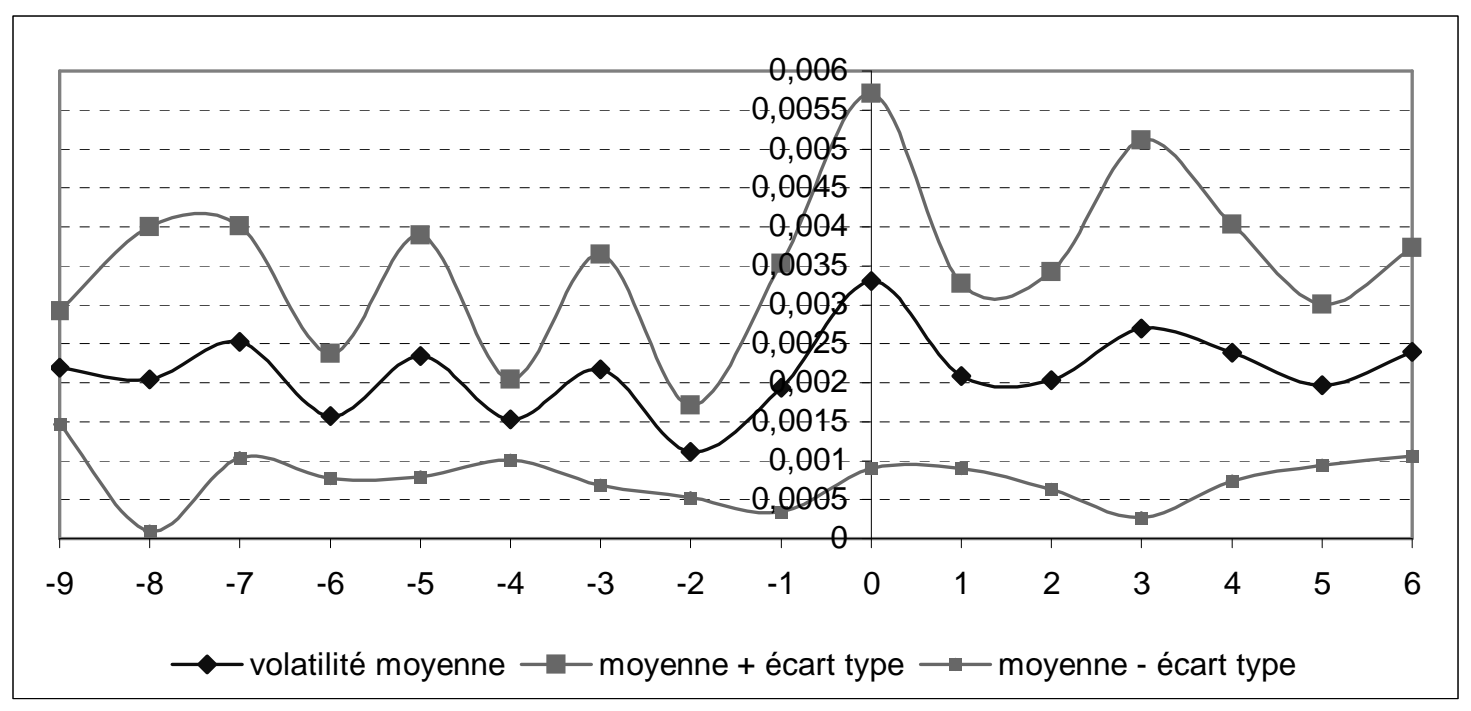

L'étude individuelle de chaque entreprise ne permet pas de tirer de conclusions quant à l'impact des réservations sur la volatilité. En effet, on ne peut tester l'efficacité des réservations en les considérant une par une. Ce n'est pas représentatif (cf. Michalon (2001)). Il est plus intéressant de faire une moyenne et donc de procéder à une étude globale. On considère la date 0 , comme le jour de la réservation. Les volatilités sont calculées avec au moins quatre entreprises. Ceci étant, nous avons pu encadrer la réservation 9 séances avant et 6 séances après. Si on considère l'intervalle $[-6,+6]$, la volatilité après réservation $(0.002259)$ est plus élevée que la volatilité avant l'interruption (0.001777).

\footnotetext{
${ }^{4}$ Le 7 , le 14 , le 21 et le 28 sont des samedis. Le $1^{\text {er }}$, le 8 , le15 et le 22 sont des dimanches.
} 


\subsection{Conclusion}

De cette étude empirique ressortent plusieurs résultats.

- Les entreprises à faible capitalisation boursière sont plus sujettes aux interruptions de cotation

- L'objectif des réservations de cotation est de guider les prix dans leur évolution et, surtout de réduire les perturbations excessives. En limitant la marge de variation par des seuils de réservation, les autorités espèrent une meilleure maitrise de la volatilité. Il apparaît clairement ici que ce n'est pas le cas car la volatilité des cours est plus élevée après réservation.

\section{Conclusion}

Aujourd'hui, les modes d'organisation des échanges sur les marchés financiers sont très variés. Les coûts et les risques supportés par les différents participants à un marché varient en fonction de l'organisation des transactions. Dans ces conditions, la structure d'un marché (son organisation institutionnelle, réglementaire et technologique) exerce une influence déterminante sur les stratégies d'offre et de demande des différentes parties prenantes au processus d'échange. De ce fait, les propriétés des prix des actifs dépendent en partie de la structure du marché sur lequel ils sont cotés. Le comportement des participants à l'échange (investisseurs/courtiers/teneurs de marché) est également influencé par la structure du marché.

Or, il n'existe pas actuellement de marché d'actions affichant en permanence (24h/24) des cours. Des mini-séances parisiennes en vigueur avant la mise en place du système CAC, aux modalités actuelles de fonctionnement des principaux marchés d'actions (NYSE, Londres, Euronext), un pas important a été franchi. Toutefois, quelle que soit la place boursière et que les cotations soient automatisées ou non, des procédures d'interruption de séances de cotation ont existé et perdurent. Le rôle annoncé des interruptions de cotation est de permettre l'information des participants et de protéger les intérêts des petits porteurs. Il s'agit donc de réduire les asymétries d'informations et la volatilité, de permettre l'émergence d'un consensus. Néanmoins, comme nous l'avons vu, la littérature théorique à ce sujet est contradictoire. 
Afin d'apporter des éléments de réponse au débat, nous avons procédé à une étude empirique du marché boursier français des actions. Nous avons mis en avant une inefficacité des réservations en terme de volatilité. Ces conclusions doivent cependant être nuancées. En effet, l'échantillon sur lequel sont basés les résultats est de petite taille ( 9 entreprises) et la période étudiée est courte (1 mois). Cette étude préliminaire est donc à prolonger dans plusieurs directions. En premier lieu, il serait intéressant de procéder à une étude globale sur les volumes. En deuxième lieu, une période d'observation plus importante pourrait permettre de tirer des conclusions. Nos recherches actuelles vont dans ce sens

\section{BIBLIOGRAPHIE}

Biais B., Foucault T., et P. Hillion, 1997, Microstructure des Marchés Financier. Institutions, modèles et tests empiriques, PUF, Collection Finance.

Brown D. P., et R.H. Jennings, 1989, "On technical analysis”, Review of Financial Studies 2, 527552.

COB, 2001, rapport annuel.

Dow J., et G. Gorton, 1989, Self-generating trade and rational fads : The response of price to new information, London Business School.

Euronext, 2001, "Les ordres de Bourse".

Euronext Paris, 2001, « Livre I - Règles de marché harmonisées ».

Greenwald B., et J. Stein, 1988, The task report : The reasoning behind the recommendations, Journal of Economic Perspectives 2, 3-23.

Grossman S.J., 1990, "Introduction to NBER Symposium on the October 1987 Crash", Review of Financial Studies 3, 1-3.

Grundy B.D., et M. McNichols, 1989, "Trade and revelation of information through prices and direct disclosure", Review of Financial Studies 2, 485-526. 
Hamon J., Marchés d'Actions : architecture et microstructure, 1995, Economica.

Jouaber K., 2000, « Gestion et réglementation du marché : le cas des interruptions de cotation », thèse de doctorat, Université Paris IX - Dauphine.

Lee, Ready et Seguin, mars 1994, « Volume, Volatility, and New York Stock Exchange Trading Halts », The journal of Finance, vol XLIX, no 1.

Mc Millan H., 1990, Circuit breakers in the S\&P500 futures market : Their effect on volatility and price discovery en Octobre 1989, Securities Exchange Commission.

Michalon K., 2001, "Impact des interruptions de cotation sur la microstructure des marchés financiers », mémoire de DEA, Université Paris X Nanterre.

MillerM. H., 1990, Volatility, episodic volatility and coordinated circuit-breakers, Université de Chicago.

ParisBourse SBF, 1998, BDM Bourse de Paris, Base de données. 